University of Nebraska - Lincoln

DigitalCommons@University of Nebraska - Lincoln

Faculty Publications, Department of Physics and Astronomy

Research Papers in Physics and Astronomy

December 2004

\title{
Quantum entanglement of anisotropic magnetic nanodots
}

\author{
Ralph Skomski \\ University of Nebraska-Lincoln, rskomski2@unl.edu \\ Andrei Y. Istomin \\ University of Nebraska-Lincoln, aistomin2@unl.edu \\ Anthony F. Starace \\ University of Nebraska-Lincoln, astarace1@unl.edu \\ David J. Sellmyer \\ University of Nebraska-Lincoln, dsellmyer@unl.edu
}

Follow this and additional works at: https://digitalcommons.unl.edu/physicsfacpub

Part of the Physics Commons

Skomski, Ralph; Istomin, Andrei Y.; Starace, Anthony F.; and Sellmyer, David J., "Quantum entanglement of anisotropic magnetic nanodots" (2004). Faculty Publications, Department of Physics and Astronomy. 17. https://digitalcommons.unl.edu/physicsfacpub/17

This Article is brought to you for free and open access by the Research Papers in Physics and Astronomy at DigitalCommons@University of Nebraska - Lincoln. It has been accepted for inclusion in Faculty Publications, Department of Physics and Astronomy by an authorized administrator of DigitalCommons@University of Nebraska Lincoln. 


\title{
Quantum entanglement of anisotropic magnetic nanodots
}

\author{
Ralph Skomski, ${ }^{1,2}$ Andrei Y. Istomin, ${ }^{1}$ Anthony F. Starace, ${ }^{1}$ and D. J. Sellmyer ${ }^{1,2}$ \\ ${ }^{1}$ Department of Physics and Astronomy, The University of Nebraska, Lincoln, Nebraska 68588-0111, USA \\ ${ }^{2}$ Center for Materials Research and Analysis, The University of Nebraska, Lincoln, Nebraska 68588-0111, USA
}

(Received 20 February 2004; published 8 December 2004)

\begin{abstract}
Anisotropic magnetic nanodots are promising physical realizations of qubits for quantum computing at finite temperature owing to their well-separated low-lying energy levels and scalability. The entanglement of two interacting magnetic nanodots is investigated and shown both analytically and numerically to be resonantly dependent on their interaction strength and on differences in their properties. These results provide criteria for fabricating and coupling magnetic nanodots so that their low-lying eigenstates can be significantly entangled.

DOI: 10.1103/PhysRevA.70.062307

PACS number(s): 03.67.Mn, 75.10.Jm, 85.35.Be
\end{abstract}

Recently [1], mesoscopic magnetic particles have been analyzed as potential qubits for quantum computing and their properties have been compared to DiVincenzo's criteria [2] for practical quantum computing devices. These nanometersized particles have large magnetic moments, making them easier to produce and to measure than other spin-based systems. They also have well-spaced energy levels owing to their intrinsic magnetocrystalline anisotropy, making them feasible for quantum computing at temperatures of the order of $10 \mathrm{~K}$. Self-assembly of magnetic nanostructures has been shown to be a viable fabrication technique [3]. In addition, networks of single-domain magnetic nanodots have been shown experimentally to be capable of performing logic operations at room temperature and to have significant advantages over current microelectronic technology [4]. While magnetic fields can be used to control the state of a single magnetic dot, pairwise entanglement of such dots is essential to construct two-qubit quantum gates [5]. However, such pairwise entanglement has not yet been analyzed in detail.

We examine here the entanglement of a pair of nanomagnets and its dependence on the magnitudes of their intrinsic spins, magnetic anisotropy parameters, and exchange coupling $J$. We show that the pairwise entanglement of ferromagnetic nanomagnets is maximal in two cases: (i) when the nanomagnets have identical properties-i.e., identical spins and magnetic anisotropies-and (ii) when their exchange coupling parameter $J$ is given by the ratio of the differences of their magnetic anisotropies to the differences of their spins. (Practical means for tuning $J$ are discussed below.) The pairwise entanglement of two mesoscopic magnets decreases signficantly when their properties do not satisfy these conditions. Such behavior is very different from the pairwise entanglement of pure Heisenberg spin systems (see, e.g., [6]).

A magnet possesses a magnetocrystalline anisotropy if its magnetic energy depends upon the angle between its magnetization direction and the crystal axes. Since the magnetocrystalline anisotropy necessarily involves spin-orbit coupling (in addition to crystal-field interactions and to interatomic exchange), one may exploit the strong spin-orbit interaction in many heavy metal atoms to tune the anisotropy over several orders of magnitude $[7,8]$. For example, the low-temperature anisotropy field of certain rare-earth compounds is about $50 \mathrm{~T}$.
We consider two nonequivalent interacting magnetic nanodots or clusters with total spins $S$ and $S^{\prime}$. Fabricated mesoscopic objects tend to have imperfections, and it is important to understand the effects of their nonequivalence on the system's quantum properties. As we shall show, small differences in the properties of the nanodots can have a major effect on the extent to which they can be entangled. The spin magnitudes $S$ and $S^{\prime}$ are proportional to the dot size and can also be written as $S=N S_{0}$ and $S^{\prime}=N^{\prime} S_{0}^{\prime}$, where $S_{0}$ and $S_{0}^{\prime}$ can be interpreted as the numbers of spins per atom and $N$ and $N^{\prime}$ as the numbers of atoms per dot. (More exactly, $S_{0}$ and $S_{0}^{\prime}$ are the spins per unit volume, while $N$ and $N^{\prime}$ are the dot volumes divided by the unit volume.)

We write the single-dot spin Hamiltonian as [7]

$$
H_{0}=-\frac{K_{u} N}{3 S^{2}}\left(3 \hat{S}_{z}^{2}-\hat{\mathbf{S}}^{2}\right)
$$

The uniaxial anisotropy constant $K_{u}$ [measured in temperature units $(\mathrm{K})$ ] reflects the chemical composition and crystal structure of the dots [9]. Since the anisotropy energy can be written as $E\left(S_{z}=0\right)-E\left(S_{z}=S\right) \equiv K_{u} N, K_{u}$ is equal to the anisotropy energy per unit volume. The energy difference between the lowest two eigenvalues of Eq. (1)-i.e., $E\left(S_{z}=S\right.$ $-1)-E\left(S_{z}=S\right)$, is $K_{u} N(2 S-1) / S^{2}$ - thus allowing one to control the energy level spacings by adjusting the dot parameter $K_{u}$. Since we consider two different dots, we must deal with two sets of parameters and unequal level spacings.

The two dots are coupled by a Heisenberg-type exchange interaction characterized by the coupling constant $J$. The total two-dot Hamiltonian is then

$$
H=H_{0}\left(K_{u}, N, \hat{\mathbf{S}}\right)+H_{0}\left(K_{u}^{\prime}, N^{\prime}, \hat{\mathbf{S}}^{\prime}\right)-J \hat{\mathbf{S}} \cdot \hat{\mathbf{S}}^{\prime} .
$$

The exchange interaction may originate from various physical mechanisms-e.g., from the net Ruderman-KittelKasuya-Yosida (RKKY) interaction with a nonferromagnetic metallic substrate or medium $[9,10]$ —or it may be realized by means of a nanojunction (e.g., a ferromagnetic wire bridging the dots and having a diameter of a few interatomic distances yields an effective exchange coupling that depends on the wire's length and cross sectional area), or as proposed in [1], by coupling neighboring magnetic dots through inductive superconducting loops, where the coupling $J$ depends on 
(a)

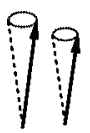

$|00\rangle$

(b)

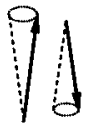

$\mid 00)$

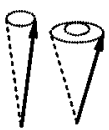

|01)

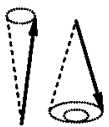

$|01\rangle$

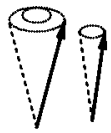

$|10\rangle$

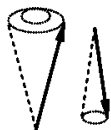

|10)

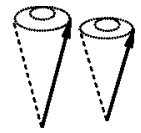

|11)

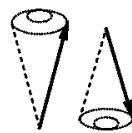

|11)
FIG. 1. Schematic of the low-lying basis states of two inequivalent nanodots coupled by (a) ferromagnetic and (b) antiferromagnetic couplings. The arrows denote single-dot spins; the $\left|S_{z}\right|=S$ states and $\left|S_{z}\right|=S-1$ states are denoted by $|0\rangle$ and $|1\rangle$, respectively.

the current induced in the loop by one spin and the field produced at the site of the other. A key point is that these means of introducing coupling allow one to control the value of $J$, which facilitates realization of two-qubit gates, as discussed in [11].

We are primarily interested in the entanglement of two interacting magnetic nanodots at relatively low temperatures as physical realizations of entangled qubits. Despite the fact that the energy spectrum of each dot comprises a ladder of states, we consider only two states per dot: the ground state $|0\rangle$ and the lowest-lying excited state $|1\rangle$. Excitations with higher energies are important at high temperatures and in some external field regimes; these corrections are estimated below and shown to be unimportant for the parameters considered here.

The physical nature of $|0\rangle$ and $|1\rangle$ depends on the values of the parameters involved. There are two main cases: ferromagnetic (FM) coupling, for which $J>0$, and antiferromagnetic (AFM) coupling, for which $J<0$. In our model, the two cases must be treated separately. For simplicity, we assume that the spin component $S_{z}$ of the first dot is positive, so that the states $|0\rangle$ and $|1\rangle$ have the quantum numbers $S_{z}=S$ and $S_{z}=S-1$, respectively. For the second dot, the respective quantum numbers are either $S_{z}^{\prime}=S^{\prime}$ and $S_{z}^{\prime}=S^{\prime}-1$ (FM case) or $S_{z}^{\prime}=-S^{\prime}$ and $S_{z}^{\prime}=-S^{\prime}+1$ (AFM case). The two-dot wave function can be written in the Schmidt basis as

$$
|\psi\rangle=\alpha|00\rangle+\beta|01\rangle+\gamma|10\rangle+\delta|11\rangle,
$$

where $|a b\rangle=|a\rangle \otimes|b\rangle$. The state $|\psi\rangle$ is pure and, in general, entangled. Well-known examples of entangled states are the four maximally entangled Bell states, which are proportional to $|00\rangle \pm|11\rangle$ and $|01\rangle \pm|10\rangle$. In Fig. 1 we illustrate the physical meaning of the quantum states for both the FM and AFM cases.

The matrix elements of the Hamiltonian in Eq. (2) in terms of the Schmidt basis are easily obtained by using the raising and lowering operators, $\hat{S}_{ \pm}=\hat{S}_{x} \pm i \hat{S}_{y}$ and $\hat{S}_{ \pm}^{\prime}=\hat{S}_{x}^{\prime} \pm i \hat{S}_{y}^{\prime}$. Taking $\langle 00|H| 00\rangle$ as our zero-point energy, the respective Hamiltonian matrices for the cases of FM and AFM couplings are

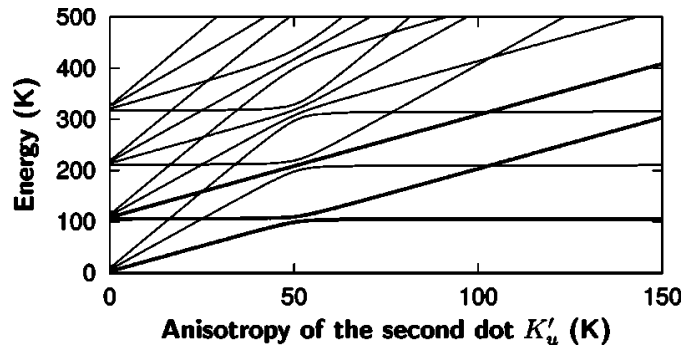

FIG. 2. Energy eigenstates of two interacting magnetic nanodots, considering, respectively, only the two and four lowestenergy levels in each dot. Curves in bold represent energy eigenvalues occurring in both the two- and four-level approximations; curves of regular thickness correspond to those occurring only in the four-level approximation. The fixed parameters are $S_{0}=S_{0}^{\prime}=1$, $N=1000, N^{\prime}=1100$, and $K_{u}=50 \mathrm{~K}$.

$$
H_{F M}=\left(\begin{array}{cccc}
0 & 0 & 0 & 0 \\
0 & Q^{\prime}+J S & -J \sqrt{S S^{\prime}} & 0 \\
0 & -J \sqrt{S S^{\prime}} & Q+J S^{\prime} & 0 \\
0 & 0 & 0 & \Sigma
\end{array}\right)
$$

and

$$
H_{A F M}=\left(\begin{array}{cccc}
0 & 0 & 0 & -J \sqrt{S S^{\prime}} \\
0 & Q^{\prime}+J S & 0 & 0 \\
0 & 0 & Q+J S^{\prime} & 0 \\
-J \sqrt{S S^{\prime}} & 0 & 0 & \Sigma
\end{array}\right) .
$$

Here $Q=K_{u} N(2 S-1) / S^{2}, Q^{\prime}=K_{u}^{\prime} N^{\prime}\left(2 S^{\prime}-1\right) / S^{\prime 2}$, and $\Sigma=Q$ $+Q^{\prime}+\left(S+S^{\prime}-1\right) J$.

The two-level approximation that we use in the present work-i.e., taking into account only the two lowest-lying levels in each of the quantum dots (so that our system has only four energy eigenstates) - requires justification. This approximation is valid provided that taking into account higher excited states of each dot does not introduce any additional energy eigenvalues of the two-dot system between the lowest-energy eigenvalues of our two-level (qubit) approximation; also, the higher excited dot states should not significantly shift the lowest eigenvalues of our two-dot system. In order to check our two-level (qubit) approximation, we have performed two additional sets of calculations, taking three and four levels into account in each of the dots. The threelevel approximation results in nine energy eigenstates, while the four-level approximation results in 16 eigenstates. In Fig. 2 we compare the calculated energy eigenvalues in the twoand four-level approximations as functions of the anisotropy parameter of the second dot, $K_{u}^{\prime}$, when the first dot has the parameter $K_{u}=50 \mathrm{~K}$. As one sees, in the range of $25 \mathrm{~K}$ $<K_{u}^{\prime}<100 \mathrm{~K}$, inclusion of higher excited dot states does not introduce any additional energy eigenvalues below the three lowest-energy eigenvalues corresponding to our two-level approximation, and all energy eigenvalues of the two-level approximation are essentially unchanged in the four-level approximation. (Similar results are found for the three-level case.) As our results below focus on the system's ground 
state and two lowest excited states, especially in the region $K_{u}^{\prime} \approx K_{u}$, the results shown in Fig. 2 justify the use of our two-level approximation for qubits.

Several measures of entanglement for two-qubit systems have been proposed and analyzed [12-16]. We use here the measure of entanglement termed concurrence [12,13,17], which is closely related to the entanglement of formation [12]. For a two-qubit system described by the density matrix $\rho$, the concurrence parameter $C$ is defined as follows $[12,17]$ :

$$
C=\max \left(\sqrt{\lambda_{1}}-\sqrt{\lambda_{2}}-\sqrt{\lambda_{3}}-\sqrt{\lambda_{4}}, 0\right),
$$

where $\lambda_{i}(i=1,2,3,4)$ are eigenvalues of the operator

$$
R=\rho\left(\sigma_{y} \otimes \sigma_{y}\right) \rho *\left(\sigma_{y} \otimes \sigma_{y}\right),
$$

which in Eq. (6) are ordered so that $\lambda_{1} \geqslant \lambda_{2} \geqslant \lambda_{3} \geqslant \lambda_{4}$. For the pure state $|\psi\rangle$ in Eq. (3), a simple expression for $C$ can be obtained [18]:

$$
C=2|\alpha \delta-\beta \gamma| .
$$

We consider first the case of FM coupling [cf. Fig. 1(a)]. The ground state $|00\rangle$ is separable. The first and second excited states are given by superpositions of two Schmidt basis states,

$$
\begin{aligned}
& \left|\psi_{1}\right\rangle=\cos \theta|01\rangle+\sin \theta|10\rangle, \\
& \left|\psi_{2}\right\rangle=\sin \theta|01\rangle-\cos \theta|10\rangle,
\end{aligned}
$$

and are generally entangled. Here $\theta$ is a mixing angle that depends on $S, S^{\prime}, Q, Q^{\prime}$, and $J$. By diagonalizing the interaction matrix $H_{F M}$, one obtains

$$
\begin{gathered}
\tan \theta=\chi-\sqrt{1+\chi^{2}}, \\
\chi \equiv \frac{Q^{\prime}-Q-J\left(S^{\prime}-S\right)}{2 J \sqrt{S S^{\prime}}} .
\end{gathered}
$$

For the states $\left|\psi_{1,2}\right\rangle$ in Eqs. (9) and (10) the concurrence has a very simple form, $C=|\sin (2 \theta)|$, which yields

$$
C=\frac{2 J \sqrt{S S^{\prime}}}{\sqrt{\left[J\left(S-S^{\prime}\right)-\left(Q-Q^{\prime}\right)\right]^{2}+4 J^{2} S S^{\prime}}} .
$$

One observes that for dots with identical properties, $C=1$. For nonequivalent dots (the typical case), nonzero values of the terms $S-S^{\prime}$ and $Q-Q^{\prime}$ in the denominator allow one to maximize the magnitude of the concurrence by adjusting the dot parameters. Specifically, for a given set of parameters $S_{0}^{\prime}=S_{0}, N, N^{\prime}=N+\Delta N, K_{u}$, and $K_{u}^{\prime}=K_{u}+\Delta K_{u}$, the value of $J$ that makes the concurrence of the first two excited states of the FM system equal to unity is

$$
J=\frac{Q-Q^{\prime}}{S-S^{\prime}}=\frac{1}{S_{0}^{2}}\left(2 \frac{\Delta K_{u}}{\Delta N}-\frac{N \Delta K_{u}-K_{u} \Delta N}{S_{0} N \Delta N(N+\Delta N)}\right) .
$$

If $(\Delta N / N) \ll 1$ and $1 /\left(2 N S_{0}\right) \ll 1$, the desired value of the coupling constant is given by

$$
J \approx \frac{1}{S_{0}^{2}}\left(2 \frac{\Delta K_{u}}{\Delta N}+\frac{1}{S_{0}} \frac{K_{u}^{\prime}}{N^{2}}\right) .
$$

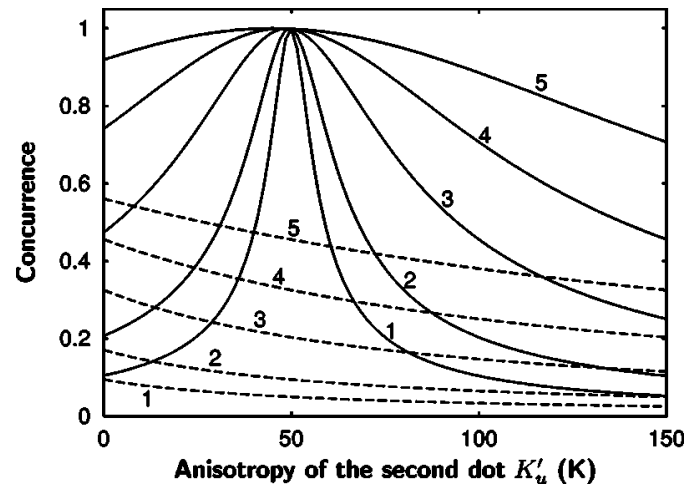

FIG. 3. Concurrence of the lowest-lying entangled states as a function of the anisotropy $K_{u}^{\prime}$ of the second dot. The solid and dashed curves denote the concurrence $C$ for the cases of FM and AFM couplings, respectively, for several values of $|J|:$ (1) $0.005 \mathrm{~K}$, (2) $0.01 \mathrm{~K}$, (3) $0.025 \mathrm{~K}$, (4) $0.05 \mathrm{~K}$, and (5) $0.1 \mathrm{~K}$. The fixed parameters are $S_{0}=S_{0}^{\prime}=1, N=1000, N^{\prime}=1100$, and $K_{u}=50 \mathrm{~K}$. A resonancelike behavior of concurrence is seen for small values of $J$ for the case of FM coupling, in contrast to the case of AFM coupling.

In the antiferromagnetic case, Fig. 1(b), the ground state is $|00\rangle$ with some admixture of $|11\rangle$ and is thus generally entangled, whereas the first two excited states are the unperturbed separable states $|01\rangle$ and $|10\rangle$. The concurrence of the AFM ground state is

$$
C=\frac{2|J| \sqrt{S S^{\prime}}}{\sqrt{\Sigma^{2}+4 J^{2} S S^{\prime}}} .
$$

One sees that $C$ decreases monotonically as the anisotropy parameter $K_{u}^{\prime}$ increases (owing to the dependence of $\Sigma$ on $\left.K_{u}^{\prime}\right)$.

Figure 3 shows the concurrence $C$ as a function of the anisotropy parameter $K_{u}^{\prime}$ of the second dot for the FM $(J$ $>0)$ and AFM $(J<0)$ couplings, for several values of $|J|$. Figure 3 shows that the larger the value of $J$, the greater is the entanglement as measured by the concurrence. However, because large couplings may lead to significant decoherence effects (as discussed below), experimental realizations are restricted to employing only small values of $J$. As Fig. 3 shows, for small values of $J$ only the FM coupling results in substantially entangled states. In fact, the FM entanglement exhibits a resonancelike behavior with its peak located in the vicinity of the avoided crossing (near $K_{u}^{\prime} \approx 50 \mathrm{~K}$ ) between the second and third energy levels shown in Fig. 2. The width of the peak depends on the interaction strength: as the value of the exchange parameter $J$ decreases, the peak narrows. For small values of $J$, a key implication of Fig. 3 is that in order to realize significant entanglement of two FM nanodots one must be able to fabricate them with similar properties. Maximally entangled states may be achieved in either of two cases: (i) the dots must be fabricated with identical properties or (ii) the properties of the fabricated dots (i.e., $N, N^{\prime}, K_{u}, K_{u}^{\prime}$, and $J$ ) must satisfy the resonance condition in Eq. (14). By appropriately tuning $J$, realization of maximum entanglement for dots fabricated with similar but not necessarily identical properties should be possible. In 
contrast to the FM case, the concurrence of the ground state in the AFM case exhibits a monotonic decrease with $K_{u}^{\prime}$, as shown by the dashed lines in Fig. 3.

Strong interdot exchange (i.e., large $J$ ) is undesirable for two reasons. First, since dynamical manipulation of entangled qubits may be realized using relatively weak timedependent magnetic fields (which are not considered here), the interdot coupling strength should be comparable to the magnitude of the interaction with such fields. Second, strong interdot coupling may lead to spin flips inside the dots so that $S$ and $S^{\prime}$ are no longer good quantum numbers, thereby invalidating our model.

The intradot exchange coupling, however, should be sufficiently strong to ensure that the single-dot quantum numbers $S$ and $S^{\prime}$ are well defined. This requires the suppression of multiplet excitations (spin waves) inside the dots. The energies of the lowest-lying spin-wave states scale as $A a^{3} / L^{2}$, where $A$ is the exchange or spin-wave stiffness [7] of the dot material, $a$ is the interatomic distance, and $L$ is the dot size. For typical materials [19,20] and for temperatures significantly higher than $10 \mathrm{~K}$, the maximum dot size should therefore not exceed a few nanometers. In larger dots, lowlying spin-wave excitations have energies comparable to the eigenvalues of $H_{F M}$ and $H_{A F M}$ in Eqs. (4) and (5).

The maximum temperature at which the magnetic dots can be used as qubits is governed by the energy level spacings of the dots. These in turn are determined by $K_{u}$ and $K_{u}^{\prime}$.
An upper limit for the achievable temperature is several tens of kelvins in high anisotropy systems with $K_{u}>10 \mathrm{MJ} / \mathrm{m}^{3}$. Such high anisotropies can be realized by exploiting the strong spin-orbit coupling of $4 d, 5 d$, and $4 f$ electrons, both in bulk $[7,20]$ and in nanostructures $[20,21]$. Note that the typical $3 d$ anisotropies (exploited in [22]) are much smaller: about 0.05 and $0.5 \mathrm{MJ} / \mathrm{m}^{3}$ for Fe and Co, respectively.

In summary, our calculations indicate that magnetocrystalline anisotropy and the Heisenberg exchange interaction can be used to realize significantly entangled states of mesoscopic ferromagnetic dots at relatively high temperatures. The nanodots can be produced by advanced processing methods, and the dot parameters, such as size, magnetocrystalline anisotropy, and interdot coupling, can be used to control the entanglement. Explicit expressions for the entanglement have been presented, and the conditions for obtaining maximal entanglement have been specified. The effects of an external magnetic field will be presented elsewhere.

This work has been supported in part by the Army Research Office, the Department of Energy (Division of Chemical Sciences, Geosciences, and Biosciences and Division of Materials Science), the W. M. Keck Foundation, the National Science Foundation Materials Research Science and Engineering Center Program, and the Nebraska Research Initiative.
[1] J. Tejada, E. M. Chudnovsky, E. del Barco, J. M. Hernandez, and T. P. Spiller, Nanotechnology 12, 181 (2001).

[2] D. P. DiVincenzo, in Mesoscopic Electron Transport, edited by L. Kowenhoven, G. Schon, and L. Sohn (Kluwer, Dordrecht, Boston, 1997).

[3] C. Yu, D. Li, J. Pearson, and S. D. Bader, Appl. Phys. Lett. 79, 3848 (2001).

[4] R. P. Cowburn and M. E. Welland, Science 287, 1466 (2000).

[5] S. Lloyd, Phys. Rev. Lett. 75, 346 (1995); A. Barenco et al., Phys. Rev. A 52, 3457 (1995).

[6] G. L. Kamta and A. F. Starace, Phys. Rev. Lett. 88, 107901 (2002), and references therein.

[7] R. Skomski and J. M. D. Coey, Permanent Magnetism (Institute of Physics, Bristol, 1999).

[8] R. M. White, Quantum Theory of Magnetism (Springer-Verlag, Berlin, 1983).

[9] R. Skomski, Europhys. Lett. 48, 455 (1999).

[10] N. J. Craig et al., Science 304, 565 (2004).

[11] N. Schuch and J. Siewert, Phys. Rev. A 67, 032301 (2003).
[12] C. H. Bennett, D. P. DiVincenzo, J. A. Smolin, and W. K. Wootters, Phys. Rev. A 54, 3824 (1996).

[13] W. K. Wootters, Phys. Rev. Lett. 80, 2245 (1998).

[14] V. Vedral, M. B. Plenio, M. A. Rippin, and P. L. Knight, Phys. Rev. Lett. 78, 2275 (1997).

[15] M. Horodecki, P. Horodecki, and R. Horodecki, Phys. Rev. Lett. 80, 5239 (1998).

[16] E. M. Rains, Phys. Rev. A 60, 173 (1999); 60, 179 (1999).

[17] S. Hill and W. K. Wootters, Phys. Rev. Lett. 78, 5022 (1997).

[18] D. Gunlycke, V. M. Kendon, V. Vedral, and S. Bose, Phys. Rev. A 64, 042302 (2001).

[19] R. Skomski, A. Kashyap, Y. Qiang, and D. J. Sellmyer, J. Appl. Phys. 93, 6477 (2003).

[20] R. Skomski, J. Phys.: Condens. Matter 15, R841 (2003).

[21] Y. C. Sui, R. Skomski, K. D. Sorge, and D. J. Sellmyer, Appl. Phys. Lett. 84, 1525 (2004).

[22] S. Guéron, M. M. Deshmukh, E. B. Myers, and D. C. Ralph, Phys. Rev. Lett. 83, 4148 (1999). 